\title{
Nuclear Energy Spectrum Decomposition Based on Hybrid Particle Swarm Optimization
}

\author{
Xing-Ke $\mathrm{Ma}^{1} \mathrm{Q}$, Yang-Zhen $\mathrm{Ji}^{1}$
}

${ }^{1}$ College of Nuclear Technology and Automation Engineering, Chengdu University of Technology, Dongsanlu, Erxianqiao, Chengdu 610059, China

\begin{abstract}
A nonlinear fitting model is proposed for the problem of nuclear energy spectrum decomposition. And the hybrid particle swarm optimization algorithm based on natural selection idea and random inertia weight is used to solve. First, a nonlinear fitting model was introduced. Secondly, the defects of the traditional particle swarm optimization algorithm based on linear inertia weight are analyzed, and the ideas of stochastic inertia weight and natural selection are integrated into the algorithm for these shortcomings. Then, according to the specific problems involved in this paper and the existing data, the continuous function model is transformed into a discrete series model. According to the nature that the absolute value is not less than zero, the fitness value is appropriately modified to achieve the purpose of improving the calculation accuracy and the operation speed of the algorithm.
\end{abstract}

Keywords: Energy Spectrum Decomposition, Nonlinear Fitting Model, Hybrid Particle Swarm

\section{Introduction}

Particle swarm optimization (PSO) simulates the process of foraging for birds, each bird representing a particle, which is also a possible solution to the problem [1-3]. Then, update the algorithm by updating the extremum: first is the optimal solution found by the particle itself, ie the individual extremum. The other extreme value is the optimal solution currently found by the entire population, namely the global extremum.[4-6]

The traditional particle swarm optimization (PSO) performs extreme value optimization through individual extremum and group extremum. [7] It has the advantages of fast search speed, simple principle and easy operation. However, as with most optimization algorithms, it is easy to fall into the local optimal solution and cannot jump out.[8, 9] Secondly, in the adjustable parameters of the PSO, the processing of the inertia weight $\omega$ plays an important role in the final calculation of the entire algorithm. Increasing the value of $\omega$ can improve the global search ability of the algorithm, and reducing the value of $\omega$ can improve the local search ability of the algorithm. Therefore, designing a reasonable value of $\omega$ is the key to avoiding PSO falling into local optimum and improving search efficiency.[10-12]

\section{Principle and algorithm design \\ 2.1 Mathematical model}

A nonlinear fitting model of the decomposition of overlapping spectral peaks is proposed, which is expressed as follows:

$\mathrm{G}(x)=\left|F(x)^{2}-\left[\sum_{i=1}^{M} a_{i} f_{i}(x)\right]^{2}\right|$
Where: $F(x)$ represents the initial mixed full spectrum of various elements, and $a_{i}$ represents the weight of the $i$-th element in the full spectrum, and satisfies:

$\sum_{i=1}^{M} a_{i}=1, \quad a_{i} \geq 0$

$M$ represents the number of elements participating in the mixing, and $f_{i}(x)$ represents the energy spectrum of the $i$-th element.

\subsection{Random inertia weight}

The inertia weighting factor $\omega$ is set to a random number obeying a normal distribution. The advantages of this approach are:

If the particles can find a better and feasible solution in the initial stage, according to the characteristics of the normal distribution, the randomly generated $\omega$ may be a relatively small value, thereby speeding up the convergence of the algorithm. Second, it can overcome the best limitation that the algorithm caused by the $\omega$ linear decrement can not converge. 服 The inertia weighting factor obeying the normal distribution can be described by the following formula[12, 13]:

$\left\{\begin{array}{c}\omega=\mu+\delta \cdot N(0,1) \\ \mu=\mu_{\text {min }}+\left(\mu_{\text {max }}-\mu_{\text {min }}\right) \cdot \operatorname{rand}(0,1)\end{array}\right.$

Where $N(0,1)$ represents a random number obeying the standard normal distribution, and $\mu_{\max }$ and $\mu_{\min }$ respectively represent the upper and lower limits of the parameter $\mu$ of the normal distribution. $[13,14]$

\subsection{Particle Swarm Optimization Algorithm Based on Genetic Algorithm}

The PSO algorithm is simple and easy to implement, 
and does not need to adjust too many parameters. [15]Although the convergence speed is fast in the early stage, it is affected by the random oscillation phenomenon in the later stage. This shortcoming makes it take a long time to search near the global optimal solution, which makes it easy to fall into the local minimum, the accuracy is reduced, and it is easy to diverge. The genetic algorithm has the advantage of strong global search ability. [16]Therefore, this paper introduces the natural selection idea in the traditional particle swarm optimization algorithm to improve the PSO algorithm.[17] In order to better decompose the energy spectrum

In each iteration, the particle swarms are sorted according to the particle swarm fitness value, replacing the worst half of the particles with the best half of the population. At the same time, the historical optimal value remembered by each individual is preserved, thereby improving the global search ability of the PSO algorithm.[18]

In summary, the introduction of random inertia weights and natural selection ideas in genetic algorithms into the PSO algorithm will help to improve the defects of PSO.

\subsection{Hybrid Particle Swarm Optimization Algorithm Based on Natural Selection and Random Inertia Weight}

The hybrid particle swarm algorithm based on natural selection and random inertia weighting is as follows [7, 11, 16, 17, 19-22]:

A. Randomly set the speed and position of each particle.

Calculate the fitness value of each particle, and store the position and fitness value of the particle in the individual extreme value $p_{\text {best }}$ of the particle.

The individual position and fitness values of the optimal fitness values in all $p_{\text {best }}$ are stored in the global optimal value $g_{\text {best }}$.

B. Update the speed and position of the particles

$$
\begin{aligned}
& V_{i, j}(t+1)=\omega V_{i, j}(t)+c_{1} r_{1}\left[p_{i, j}-x_{i, j}(t)\right]+ \\
& c_{2} r_{2}\left[p_{g, j}-x_{i, j}(t)\right](4) \\
& X_{i, j}(t+1)=X_{i, j}(t)+\beta V_{i, j}(t+1)(5)
\end{aligned}
$$

Where, $V_{i, j}(t+1), X_{i, j}(t+1)$ represents the velocity and position of the $i$-th particle in the $j$-th dimension in the $t+1$-th iteration.

$p_{i, j}, p_{g, j}$ respectively represent the global optimal value of the individual optimal value of the $i$-th particle at the end of the t-th iteration.

$c_{1}, c_{2}$ are learning factors, also called acceleration constants. $r_{1}, r_{2}$ is a uniform random number in the range $[0,1] . \beta$ is called the constraint factor and is used to adjust the weight, $\omega$ is the inertia weight.

C. Update weights by random weight method
The fitness value of each particle is compared to the best position of the particle. If it is similar, the current value is taken as the best position of the particle. Compare all current $p_{\text {best }}$ and $g_{\text {best }}$, update $g_{\text {best }}$

Sort the particle swarms based on fitness values, replacing the worst half of the particles with the best half of the population, while preserving the historical best values remembered by each individual.[23-25]

When the algorithm reaches the stop condition, the search is stopped and the result is output; otherwise, return to step $\mathrm{C}$ to continue the search.

The algorithm flow chart is shown below:

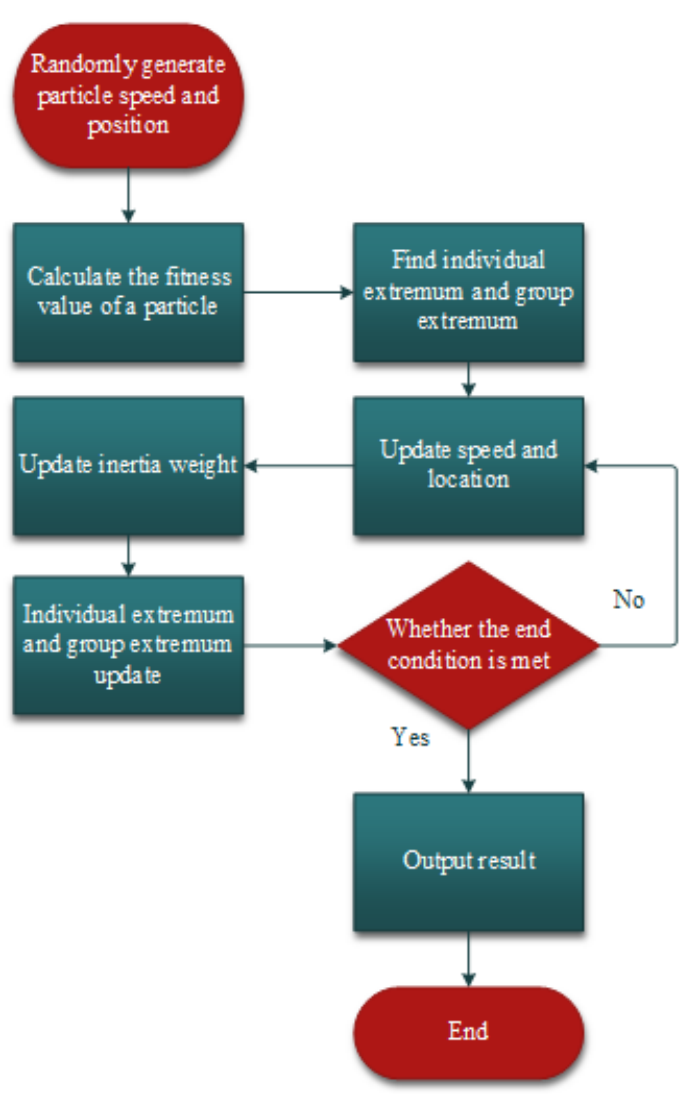

Fig .1 Algorithm flowchart

\section{Examples}

The hybrid particle swarm optimization algorithm based on natural selection and random inertia weight also has good precision for overlapping spectral peak decomposition in $\mathrm{X}$-spectrum analysis. Due to the analysis of overlapping spectral peaks, there are still a series of difficulties such as real-time processing difficulties and convergence to local optimal solutions. At present, only a certain mathematical model can be used to transform this problem, so as to achieve the purpose of spectrum dissociation to the utmost extent. $\mathrm{Xi}$ Yang et al.[14] proposed a method for analyzing overlapping peaks based on Gaussian Mixture Model - Standard Deviation Related, (GMM-SDR) of particle swarm optimization, and obtained high precision results. However, due to the defects of the 
traditional particle swarm algorithm, when the positions of the peaks are close to each other, or the area of the peaks differs greatly, the method may have a large error. The hybrid particle swarm optimization algorithm based on natural selection and random inertia weight proposed in this paper can improve the above problems to some extent.

\subsection{GMM-SDR model}

According to Hong-Quan Huang[26] et al., Gaussian Mixture Model-Standard Deviation Related , (GMM-SDR):

$P(x \mid \theta)=\sum_{i=1}^{M} a_{i} \frac{1}{\sqrt[2]{2 \pi} f_{\sigma}(i)} e^{-\frac{\left(x-u_{i}\right)^{2}}{2 f_{\sigma}(i)^{2}}}$

Where: $a_{i}$ represents the weight of the $i$-th peak and satisfies:

$\sum_{i=1}^{M} a_{i}=1, a_{i} \geq 0$

$u_{i}, f_{\sigma}(i)$ are the mean and standard deviation of the $i$-th peak, respectively, which are linearly distributed in this paper. This correlation is reflected in the correlation between the standard deviations between the peaks, where $f_{\sigma}(i)=u_{1} f_{\sigma}(1) / u_{1}(i=2,3 \ldots, M)$, 对 The parameters for the above GMM-SDR model can be expressed as:

$\theta=\left[\begin{array}{c}a_{1}, a_{2}, \ldots, a_{M} \\ u_{1}, u_{2}, \ldots, u_{M} \\ f_{\sigma}(1), f_{\sigma}(2) \ldots f_{\sigma}(M)\end{array}\right]$

Parameter estimation method for the GMM-SDR model. First, a hybrid particle swarm optimization algorithm based on natural selection and random inertia weights is set. Secondly, the probability that the random number $x(1), x(2), \ldots, x(\mathrm{~N})$ forming the original overlapping peak in the statistical sense is attributed to each GMM-SDR model is calculated. Finally, using the searched "global maximum probability" position information parameter $\theta$ as the final solution, the weight, mean and standard deviation of each peak of the overlapping spectrum can be obtained.

\subsection{Spectral decomposition with large difference in peak area}

As shown in Figure 2, the overlap spectrum of the setup experiment was formed by three Gaussian peaks with peak positions of 180, 190, and 205 and peak areas of 1500,15000 , and 15000 , respectively.

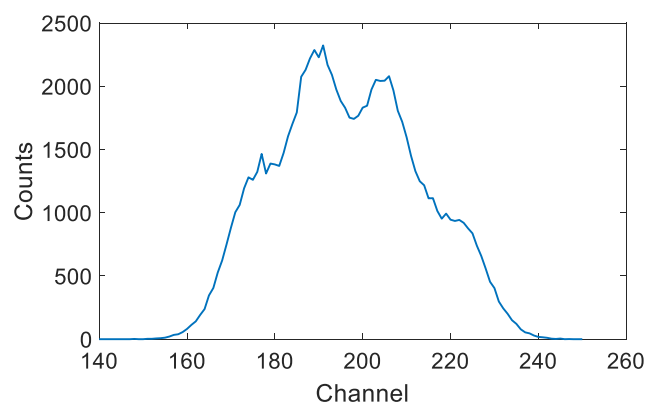

Fig. 2 Overlapping peak

Set the population to 50 and the initialization range to
[0.01 0.010 .011601601603 ; 111122252252258 . The number of iterations $\mathrm{T}=200, c_{1}=c_{2}=1.49445$. The constraint factor $\beta=0.5, \omega_{\max }=0.9, \omega_{\min }=$ 0.4 。 According to the traditional linear inertia weight particle swarm optimization algorithm, the image after decomposing the energy spectrum It can be seen intuitively that the energy spectrum with smaller peak area can not be solved, and the decomposition spectrum fails.

The effect of the decomposition energy spectrum of the particle swarm optimization algorithm based on natural selection thought provided in this paper is as follows:

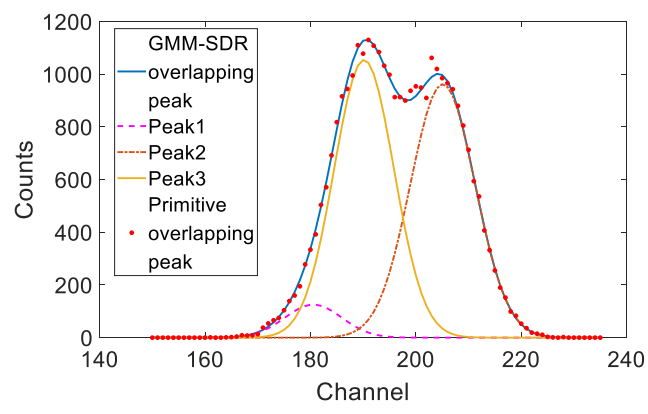

Fig.3 Decomposed peak

Original spectrum, GMM-SDR

Peak of curve and decomposition

Perform error analysis on the parameters after decomposing the energy spectrum, as shown in Table 1

Table 1 error analysis

\begin{tabular}{|c|c|c|c|}
\hline & Raw data & $\begin{array}{c}\text { The } \\
\text { Result of } \\
\text { GMM-S } \\
\text { DR }\end{array}$ & $\begin{array}{c}\text { Relative } \\
\text { error } \\
\%\end{array}$ \\
\hline$a_{1}$ & $4.76 \%$ & $4.35 \%$ & 8.61 \\
\hline$a_{2}$ & $47.62 \%$ & $47.91 \%$ & 0.61 \\
\hline$a_{3}$ & $47.62 \%$ & $47.74 \%$ & 0.25 \\
\hline$u_{1}$ & 180 & 179.94 & 0.11 \\
\hline$u_{2}$ & 190 & 190.03 & 0.21 \\
\hline$u_{3}$ & 205 & 205.09 & 0.01 \\
\hline$f_{\sigma}(1)$ & 5.4 & 5.404 & 0.07 \\
\hline$f_{\sigma}(2)$ & 5.7 & 5.603 & 0.17 \\
\hline$f_{\sigma}(3)$ & 6.15 & 6.132 & 0.29 \\
\hline
\end{tabular}

\section{Conclusion}

In the energy spectrum decomposition problem, the particle swarm optimization algorithm based on natural selection and stochastic inertia weight can realize the dissociation target more quickly and more accurately than the traditional linear inertia weight particle swarm optimization algorithm. Although the complex spectrum of the three peaks with large difference in decomposition peak area is high, the hybrid particle swarm optimization algorithm based on natural selection proposed in this paper still has a good effect on solving this problem. 


\section{References}

1. C. Trelea, "The particle swarm optimization algorithm: convergence analysis and parameter selection," Information Processing Letters, vol. 85, no. 6, pp. 317-325, Mar 31 2003, Art. no. Pii s0020-0190(02)00447-7.

2. Z. L. Gaing, "Particle swarm optimization to solving the economic dispatch considering the generator constraints," Ieee Transactions on Power Systems, vol. 18, no. 3, pp. 1187-1195, Aug 2003.

3. A. C. Coello, G. T. Pulido, and M. S. Lechuga, "Handling multiple objectives with particle swarm optimization," Ieee Transactions on Evolutionary Computation, vol. 8, no. 3, pp. 256-279, Jun 2004.

4. J. Robinson and Y. Rahmat-Samii, "Particle swarm optimization in electromagnetics," Ieee Transactions on Antennas and Propagation, vol. 52, no. 2, pp. 397-407, Feb 2004.

5. F. van den Bergh and A. P. Engelbrecht, "A cooperative approach to particle swarm optimization," Ieee Transactions on Evolutionary Computation, vol. 8, no. 3, pp. 225-239, Jun 2004.

6. Z. L. Gaing, "A particle swarm optimization approach for optimum design of PID controller in AVR system," Ieee Transactions on Energy Conversion, vol. 19, no. 2, pp. 384-391, Jun 2004.

7. F. Juang, "A Hybri of genetic algorithm and particle swarm optimization for recurrent network design," Ieee Transactions on Systems Man and Cybernetics Part B-Cybernetics, vol. 34, no. 2, pp. 997-1006, Apr 2004.

8. B. Liu, L. Wang, Y. H. Jin, F. Tang, and D. X. Huang, "Improved particle swarm optimization combined with chaos," Chaos Solitons \& Fractals, vol. 25, no. 5, pp. 1261-1271, Sep 2005.

9. J. B. Park, K. S. Lee, J. R. Shin, and K. Y. Lee, "A particle swarm optimization for economic dispatch with nonsmooth cost functions," Ieee Transactions on Power Systems, vol. 20, no. 1, pp. 34-42, Feb 2005.

10. J. J. Liang, A. K. Qin, P. N. Suganthan, and S. Baskar, "Comprehensive learning particle swarm optimizer for global optimization of multimodal functions," Ieee Transactions on Evolutionary Computation, vol. 10, no. 3, pp. 281-295, Jun 2006.

11. Y. del Valle, G. K. Venayagamoorthy, S. Mohagheghi, J.-C. Hernandez, and R. G. Harley, "Particle swarm optimization: Basic concepts, variants and applications in power systems," Ieee Transactions on Evolutionary Computation, vol. 12, no. 2, pp. 171-195, Apr 2008

12. Z.-H. Zhan, J. Zhang, Y. Li, and H. S.-H. Chung, "Adaptive Particle Swarm Optimization," Ieee Transactions on Systems Man and Cybernetics Part B-Cybernetics, vol. 39, no. 6, pp. 1362-1381, Dec 2009.

13. Y. Wang, J. Lv, L. Zhu, and Y. Ma, "Crystal structure prediction via particle-swarm optimization," Physical Review B, vol. 82, no. 9, Sep 28 2010, Art. no. 094116.

14. X. Yang, H.-q. Huang, K.-m. Jiang, W.-d. Chen, W. Zhou, and M.-m. Wang, "Application of Particle Swarm Algorithm and
GMM-SDR Model in Overlapping Spectrum Peak Analysis," Spectroscopy and Spectral Analysis, vol. 37, no. 8, pp. 2376-2380, Aug 2017.

15. L. I. U. Dong, H. A. O. Ting, and L. I. U. Xiyu, "Cauchy Particle Swarm optimization based on dynamic probability mutation," Computer Engineering and Application, vol. 43, no. 16, pp. 77-79, 2007 2007, Art. no. 1002-8331(2007)43:16<77:Jydtgl>2.0.Tx;2-j.

16. Y. Yaping, T. A. N. Ying, and Z. Jianchao, "Quadratic Particle Swarm Optimization and its Self-Adaptive Parameters," Computer Engineering and Application, vol. 42, no. 31, pp. 64-67, 2006 2006, Art. 1002-8331(2006)42:31<64:Ecwlqs >2.0.Tx;2-d

17. K. Yan, F. Hai-peng, X. U. Wen-bo, and Y. Yan-ping, "Cooperative approach to Quantum-behaved Particle Swarm Optimization," Computer Engineering and Application, vol. 46, no. 4, pp. 39-42,112, 2010 2010, Art. no. 1002-8331(2010)46:4<39:Hzdjyl>2.0.Tx;2-x.

18. Li, H. Guo, L. Liu, and W. Li, "Resolving vehicle routing problem with improved sweep-particle swarm optimization algorithm," Computer Engineering and Application, vol. 48, no. 20, pp. 216-223, $2012 \quad 2012$, Art. no. 1002-8331(2012)48:20<216:Gjxlzq>2.0.Tx;2-a.

19. J. Grobler and A. P. Engelbrecht, "Arithmetic and parent-centric headless chicken crossover operators for dynamic particle swarm optimization algorithms," Soft Computing, vol. 22, no. 18, pp. 5965-5976, Sep 2018.

20. K. Deb, "An efficient constraint handling method for genetic algorithms," Computer Methods in Applied Mechanics and Engineering, vol. 186, no. 2-4, pp. 311-338, 20002000.

21. K. Deb, A. Pratap, S. Agarwal, and T. Meyarivan, "A fast and elitist multiobjective genetic algorithm: NSGA-II," Ieee Transactions on Evolutionary Computation, vol. 6, no. 2, pp. 182-197, Apr 2002, Art. no. Pii s 1089-778x(02)04101-2.

A. Konak, D. W. Coit, and A. E. Smith, "Multi-objective optimization using genetic algorithms: A tutorial," Reliability Engineering \& System Safety, vol. 91, no. 9, pp. 992-1007, Sep 2006.

22. Rudolph, "Convergence analysis of canonical genetic algorithms," IEEE transactions on neural networks, vol. 5, no. 1, pp. 96-101, 19941994.

23. Jones, P. Willett, and R. C. Glen, "Molecular recognition of receptor sites using a genetic algorithm with a description of desolvation," Journal of molecular biology, vol. 245, no. 1, pp. 43-53, 1995-Jan-06 1995.

24. S. A. Kazarlis, A. G. Bakirtzis, and V. Petridis, "A genetic algorithm solution to the unit commitment problem," Ieee Transactions on Power Systems, vol. 11, no. 1, pp. 83-90, Feb 1996.

25. H.-q. Huang, W.-c. Ding, D.-c. Gong, and F. Fang, "Decomposition of X-Ray Fluorescence Overlapping Peaks Based on Statistical and Genetic Algorithms," Spectroscopy and Spectral Analysis, vol. 35, no. 8, pp. 2320-2323, Aug 2015. 